\title{
Vibration-based crack diagnosis in rotating shafts during acceleration through resonance
}

\author{
Jerzy T. Sawicki ${ }^{* 1}$, Xi Wu*, George Y. Baaklini ${ }^{\dagger}$, Andrew L. Gyekenyesi ${ }^{* *}$ \\ "Cleveland State University, Dept. of Mechanical Engineering, Cleveland, OH 44115; \\ †NASA Glenn Research Center, 21000 Brookpark Road, MS 6-1, Cleveland, OH 44135; \\ ** OAI/NASA Glenn Research Center, 21000 Brookpark Road, MS 6-1, Cleveland, OH 44135
}

\begin{abstract}
The dynamic response of a cracked Jeffcott rotor passing through the critical speed with constant acceleration is investigated analytically and numerically. The nonlinear equations of motion are derived and include a simple hinge model for small cracks and Mayes' modified function for deep cracks. The equations of motion are integrated in the rotating coordinate system. The angle between the crack centerline and the shaft vibration (whirl) vector is used to determine the closing and opening of the crack, allowing one to study the dynamic response with and without the rotor weight dominance. Vibration phase response is used as one of possible tools for detecting the existence of cracks. The results of parametric studies of the effect of crack depth, unbalance eccentricity orientation with respect to crack, and the rotor acceleration on the rotor's response are presented.
\end{abstract}

Keywords: crack, rotor, diagnosis, acceleration, resonance, unbalance, nonsynchronous response.

\section{INTRODUCTION}

In recent years, there has been an increasing interest for the development of rotating machinery shaft crack detection methodologies and on-line techniques ${ }^{1-9}$. Shaft crack problems present a significant safety and loss hazard in nearly every application of modern turbomachinery, particularly in the power generation industry, but not only. Recently, the Federal Aviation Administration (FAA), following several reports of shaft cracks, called $^{10}$ for additional NDE inspections of certain main rotor shafts in helicopters. Conventional NDE methods, however, have unacceptable limits. Some of these techniques are time-consuming and inconvenient for turbomachinery service testing. Almost all of these techniques require that the vicinity of the damage is known in advance, and they can provide only local information and no indication of the structural strength at a component and/or system level. Also, the effectiveness of these experimental techniques is affected by the high measurement noise levels existing in complex turbomachine structures. Therefore, the use of vibration monitoring along with vibration analysis have recently received increasing attention.

An extensive review of the early literature on cracked shafts was published by Wauer ${ }^{11}$. Dimentberg ${ }^{3}$ was apparently the first to report the effect of the rotating asymmetry on the shaft lateral vibration. Henry and Okah-Avae ${ }^{4}$ presented the results of analog computer study and reported the subcritical resonance due to an interaction between the crack and the rotor's weight. Mayes and Davies ${ }^{5}$ studied the behavior of a cracked shaft model which took into account opening and closing of the crack as a stiffness step function. Later, they extended their work and suggested a method for calculation of change in stiffness due to a deep $\operatorname{crack}^{6}$. Gasch ${ }^{7-8}$ modeled the breathing crack by a spring-loaded hinge, and numerically demonstrated a subharmonic resonance. Grabowski ${ }^{9}$ used modal approach to the problem and demonstrated numerically strong dependence of the rotor vibrational behavior on the crack position.

The previously published research results focused primarily on the study of cracked shafts subjected to constant angular speed. However, it has been well recognized ${ }^{10}$ that vibration monitoring during startup or shutdown can reveal important

1Jerzy.T.Sawicki@grc.nasa.gov; phone 1.216.433.2467; fax 1.216.977.7150 
machine malfunctions, especially for machines such as aircraft engines, which are subjected to frequent starts and stops as well as high speeds and acceleration rates. The topic of transient cracked rotor response has been treated by only a few authors. Plaut et al. ${ }^{13}$ analyzed the transient response of a simply supported, rotating, Euler-Bernoulli shaft with a breathing transverse crack, during its passage through a critical speed at constant acceleration or deceleration. Recently Sekhar ${ }^{14-15}$ investigated the transient vibration response of a cracked rotor passing through its critical speed, utilizing a simple hinge model for small cracks. He made an assumption that the vibrations remain small in comparison to the sag of the rotor under its own weight. If a cracked shaft rotates slowly under the load of its own weight, then the crack will open and close once per revolution. He noted oscillations which are developed near critical speed.

In this paper, the Jeffcott rotor model is analyzed and a transverse crack is assumed at the middle of the shaft. The characteristic changes in the accelerating rotor phase response due to the crack and are presented as a possible diagnostic tool for crack detection in the accelerated rotors. The criterion for the opening and closing of the crack has been developed based on the angle between the crack centerline and the shaft vibration vector which makes possible one to ignore the rotor weight-dominance assumption. Stiffness weakening effects of cracked rotor in both strong and weak axes are taken into consideration for deep cracks.

\section{ROTOR AND CRACK MODEL}

The theoretical model, called the Jeffcott rotor, employs a flexible rotor composed of a centrally located unbalanced disk attached to a massless elastic shaft which is, in turn, mounted symmetrically on rigid bearings (see Fig. 1(a). The shaft does have a transverse crack running across its section and located close to the disk. The stiffness of the uncracked rotor system is symmetric (isotropic) and the damping due to the air resistance effect is assumed to be viscous.

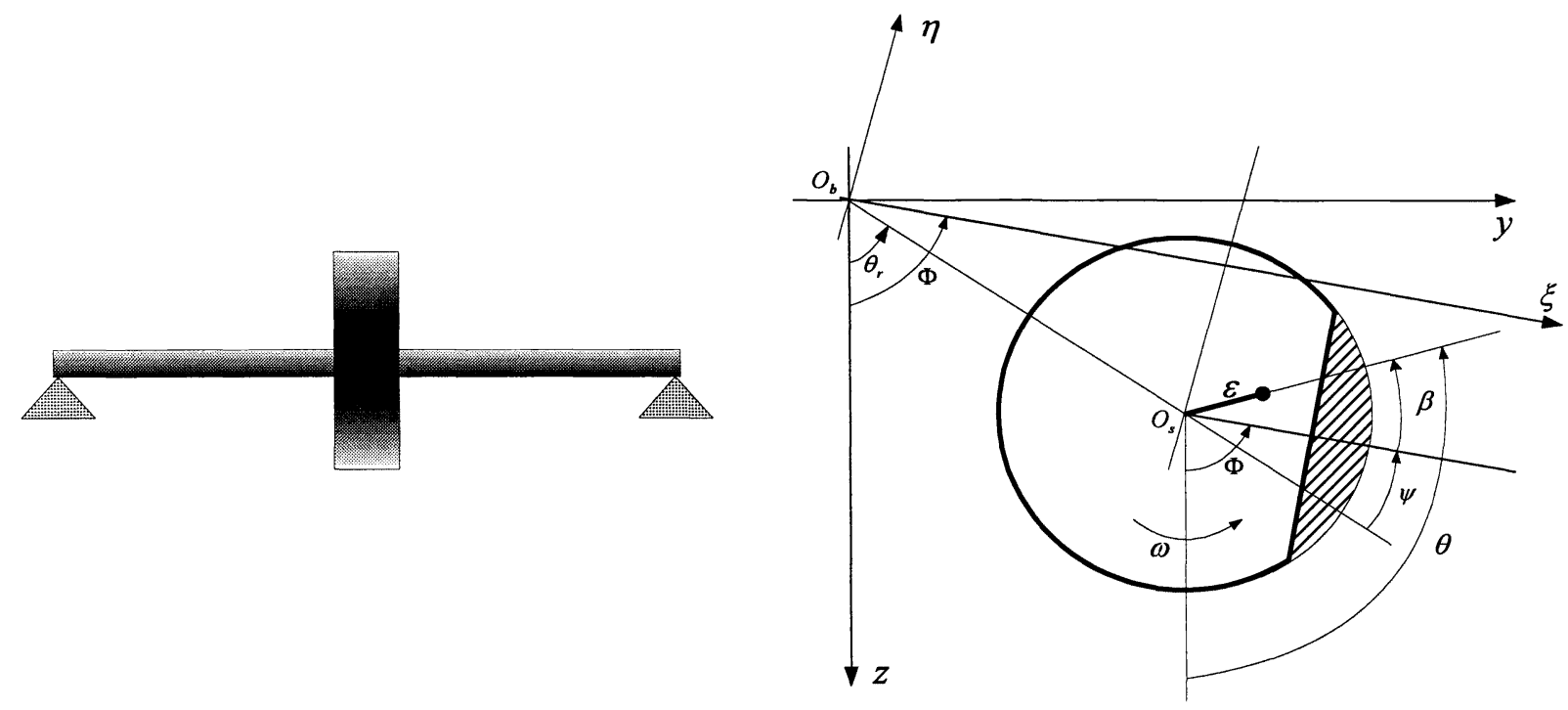

(a)

(b)

Figure 1: (a) Model of Jeffcott rotor with crack, (b) shaft and crack position described in inertial and rotating coordinate systems.

The origins of the inertial $(x, y, z)$ and body-fixed rotating $(\zeta, \eta, \xi)$ coordinate frames coincide with each other and are "attached" to the center line of the bearing supports. At any instant of time, the $\xi$-axis remains perpendicular to the face of the crack, causing that $(\zeta, \eta, \zeta)$ frame rotates with the same velocity as the rotor. The crack position with respect to the vibration vector is determined by angle $\psi$, which changes continuously with the rotor's speed. Finally, the rotor's unbalance eccentricity $\varepsilon$ is oriented at constant angle $\beta$ with respect to the crack or $\xi$-axis. For a rotor subjected to 
constant acceleration $\alpha$, the spin angle changes, i.e., $\Phi(t)=\Phi(0)+\omega_{0} t+\frac{\alpha t^{2}}{2}$, where $\omega_{0}$ is the initial speed (assumed to be zero) and $\Phi(0)=-\beta$.

The opening and closing or "breathing" of the crack, as the rotor rotates, results in a periodic change in the rotor stiffness. In order to not be restricted to the weight dominance assumption, where the dynamic displacement is smaller than the rotor's sag, the angle $\psi$ (see Fig. 1(b)) is used to judge the opening and closing of the crack. This angle more practically describes the "breath" of the crack by taking into consideration the influence of the whirl speed on the closing and opening of crack. The rotor stiffness in the crack and cross directions can be written as ${ }^{8}$ :

$$
\left(\begin{array}{cc}
K_{\xi} & 0 \\
0 & K_{\eta}
\end{array}\right)=\left(\begin{array}{cc}
K & 0 \\
0 & K
\end{array}\right)-f(\psi)\left(\begin{array}{cc}
\Delta K_{\xi} & 0 \\
0 & \Delta K_{\eta}
\end{array}\right)
$$

For linearly elastic crack and rotor deflections, the changes in the stiffness $\Delta K_{\xi}$ and $\Delta K_{\eta}$ may be assumed to be constant. For all but very large cracks, the stiffness change $\Delta K_{\eta}$ is small and often assumed to be zero. However, in this study, for the case of the deep crack, its effect is included assuming that ${ }^{6} \Delta K_{\eta}=\frac{\Delta K_{\xi}}{6}$. The steering function $f(\psi)$ is a periodic function depending on both, the rotor spin and whirl speed, and is shown on Fig. 2 for small and deep cracks.
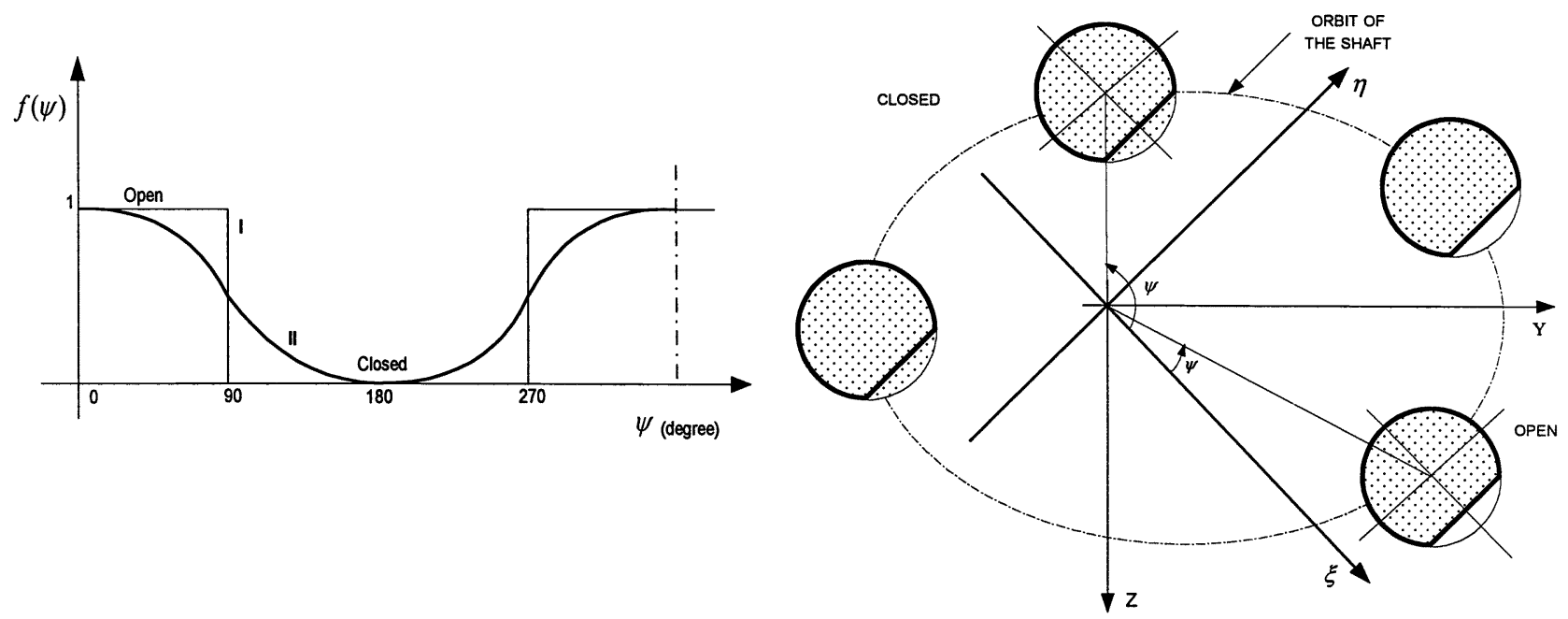

(a)

(b)

Figure 2: Crack model; (a) the closing behavior of (I) the hinge model for small cracks and (II) Mayes' modified function for deep cracks, (b) opening and closing of the crack as a function of the shaft position along its orbit.

For small cracks, the rectangular function for the hinge model $^{8}$ is used (see Fig. 2(a)), which describes the crack's sudden opening and closing, by switching from 1 (open) to 0 (closed). This function can be approximated by the Fourier expansion as follows:

$$
f(\psi)=\frac{1}{2}+\frac{2}{\pi} \cos \psi-\frac{2}{3 \pi} \cos 3 \psi+\frac{2}{5 \pi} \cos 5 \psi-\ldots
$$


In the case of deep cracks, there is a smooth transition between the opening and closing of the crack and this is reflected by the Mayes' modified function ${ }^{6}$, i.e.,

$$
f(\psi)=\frac{1+\cos \psi}{2}
$$

\section{EQUATIONS OF MOTION}

The equations of motion for a Jeffcott rotor, with a cracked shaft, in a presence of gravity forces and unbalance excitation, and subjected to constant acceleration, can be expressed in inertial coordinate frame as follows:

$$
\left(\begin{array}{cc}
M & 0 \\
0 & M
\end{array}\right)\left\{\begin{array}{l}
\ddot{z} \\
\ddot{y}
\end{array}\right\}+\left(\begin{array}{cc}
C & 0 \\
0 & C
\end{array}\right)\left\{\begin{array}{l}
\dot{z} \\
\dot{y}
\end{array}\right\}+\left(\begin{array}{ll}
K_{11} & K_{12} \\
K_{21} & K_{22}
\end{array}\right)\left\{\begin{array}{l}
z \\
y
\end{array}\right\}=\left\{\begin{array}{c}
M g \\
0
\end{array}\right\}+M \varepsilon\left\{\begin{array}{l}
\dot{\theta}^{2} \cos \theta+\ddot{\theta} \sin \theta \\
\dot{\theta}^{2} \sin \theta-\ddot{\theta} \cos \theta
\end{array}\right\}
$$

where $z$ and $y$ are the displacements of the disk. The stiffness matrix $\mathbf{K}(\mathbf{q}, t)$ (where $\mathbf{q}=\left(\begin{array}{ll}z & y\end{array}\right)^{T}$ ) is nonlinear, depending not only on time, but also on the position of the shaft center along the orbit.

The above equations of motion in the rotating coordinate can be written as

$$
\begin{aligned}
& \left(\begin{array}{cc}
M & 0 \\
0 & M
\end{array}\right)\left\{\begin{array}{l}
\ddot{\xi} \\
\ddot{\eta}
\end{array}\right\}+\left(\begin{array}{cc}
C & -2 M \omega \\
2 M \omega & C
\end{array}\right)\left\{\begin{array}{l}
\dot{\xi} \\
\dot{\eta}
\end{array}\right\}+\left(\begin{array}{cc}
K-f(\psi) \Delta K_{\xi}-M \omega^{2} & -\omega C-M \alpha \\
\omega C+M \alpha & K-f(\psi) \Delta K_{\eta}-M \omega^{2}
\end{array}\right)\left\{\begin{array}{l}
\xi \\
\eta
\end{array}\right\} \\
& =M g\left\{\begin{array}{c}
\cos \Phi \\
-\sin \Phi
\end{array}\right\}+M \varepsilon \omega^{2}\left\{\begin{array}{l}
\cos \beta \\
\sin \beta
\end{array}\right\}+M \varepsilon \alpha\left\{\begin{array}{c}
\sin \beta \\
-\cos \beta
\end{array}\right\} ;
\end{aligned}
$$

where the transformation between the inertial and rotating coordinate frames takes the form:

$$
\left(\begin{array}{l}
z \\
y
\end{array}\right)=\left(\begin{array}{cc}
\cos \Phi & -\sin \Phi \\
\sin \Phi & \cos \Phi
\end{array}\right)\left(\begin{array}{l}
\xi \\
\eta
\end{array}\right)
$$

Incorporating the hinge model for small cracks into Eq. (5) $\left(\Delta K_{\eta}=0\right)$, normalizing displacements with respect to static deflection, and using nondimensional time yields:

$$
\begin{aligned}
& {\left[\begin{array}{ll}
1 & 0 \\
0 & 1
\end{array}\right]\left\{\begin{array}{l}
\bar{\xi}^{\prime \prime} \\
\bar{\eta}^{\prime \prime}
\end{array}\right\}+\left[\begin{array}{cc}
2 \zeta & -2 \frac{\omega}{\omega_{n}} \\
2 \frac{\omega}{\omega_{n}} & 2 \zeta
\end{array}\right]\left\{\begin{array}{l}
\bar{\xi}^{\prime} \\
\bar{\eta}^{\prime}
\end{array}\right\}+\left[\begin{array}{cc}
1-f(\psi) \Delta K-\frac{\omega^{2}}{\omega_{n}^{2}} & -2 \zeta \frac{\omega}{\omega_{n}}-\frac{\alpha}{\omega_{n}^{2}} \\
2 \zeta \frac{\omega}{\omega_{n}}+\frac{\alpha}{\omega_{n}^{2}} & 1-\frac{\omega^{2}}{\omega_{n}^{2}}
\end{array}\right]\left\{\begin{array}{l}
\bar{\xi} \\
\bar{\eta}
\end{array}\right\}=} \\
& \left\{\begin{array}{c}
\cos \Phi \\
-\sin \Phi
\end{array}\right\}+\left\{\begin{array}{l}
\frac{\varepsilon}{\eta_{s t}} \frac{\omega^{2}}{\omega_{n}^{2}} \cos \beta+\frac{\varepsilon}{\eta_{s t}} \frac{\alpha}{\omega_{n}^{2}} \sin \beta \\
\frac{\varepsilon}{\eta_{s t}} \frac{\omega^{2}}{\omega_{n}^{2}} \sin \beta-\frac{\varepsilon}{\eta_{s t}} \frac{\alpha}{\omega_{n}^{2}} \cos \beta
\end{array}\right\} ;
\end{aligned}
$$

where here $f(\psi)$ is a rectangular steering function (see Fig. 2(a)). 
Similarly, one can incorporate into the equations of motion (Eq. (5)) the model for deep crack, assuming $\Delta K_{\eta}=\frac{\Delta K_{\xi}}{6}$, and Meyes ${ }^{6}$ modified steering function $f(\psi)=\frac{1+\cos (\psi)}{2}$ (see Fig. 2(a)), and write that

$$
\begin{aligned}
& {\left[\begin{array}{ll}
1 & 0 \\
0 & 1
\end{array}\right]\left\{\begin{array}{l}
\bar{\xi}^{\prime \prime} \\
\bar{\eta}^{\prime \prime}
\end{array}\right\}+\left[\begin{array}{cc}
2 \zeta & -2 \frac{\omega}{\omega_{n}} \\
2 \frac{\omega}{\omega_{n}} & 2 \zeta
\end{array}\right]\left\{\begin{array}{l}
\bar{\xi}^{\prime} \\
\bar{\eta}^{\prime}
\end{array}\right\}+\left[\begin{array}{cc}
1-f(\psi) \Delta K-\frac{\omega^{2}}{\omega_{n}^{2}} & -2 \zeta \frac{\omega}{\omega_{n}}-\frac{\alpha}{\omega_{n}^{2}} \\
2 \zeta \frac{\omega}{\omega_{n}}+\frac{\alpha}{\omega_{n}^{2}} & 1-\frac{f(\psi) \Delta K}{6}-\frac{\omega^{2}}{\omega_{n}^{2}}
\end{array}\right]\left\{\begin{array}{l}
\bar{\xi} \\
\bar{\eta}
\end{array}\right\}=} \\
& \left\{\begin{array}{c}
\cos \Phi \\
-\sin \Phi
\end{array}\right\}+\left\{\begin{array}{l}
\frac{\varepsilon}{\eta_{s t}} \frac{\omega^{2}}{\omega_{n}^{2}} \cos \beta+\frac{\varepsilon}{\eta_{s t}} \frac{\alpha}{\omega_{n}^{2}} \sin \beta \\
\frac{\varepsilon}{\eta_{s t}} \frac{\omega^{2}}{\omega_{n}^{2}} \sin \beta-\frac{\varepsilon}{\eta_{s t}} \frac{\alpha}{\omega_{n}^{2}} \cos \beta
\end{array}\right\}
\end{aligned}
$$

In the above equations the following definitions and nondimensional variables were employed (see Nomenclature section):

$$
\begin{aligned}
& \omega_{n}^{2}=\frac{K}{M}, \quad \Delta K=\frac{\Delta K_{\xi}}{K}, \quad \zeta=\frac{C}{2 M \omega_{n}}, \quad \gamma=\frac{\alpha}{\omega_{n}^{2}} \\
& \eta_{s t}=\frac{M g}{K}=\frac{g}{\omega_{n}^{2}}, \quad \bar{\xi}=\frac{\xi}{\eta_{s t}}, \quad \bar{\eta}=\frac{\eta}{\eta_{s t}}, \quad \tau=\omega_{n} t, \quad \frac{\omega}{\omega_{n}}=\gamma \tau \\
& \omega=\omega_{0}+\alpha t=\alpha t, \text { for } \omega_{0}=0 \\
& \Phi=\Phi_{0}+\omega_{0} t+\frac{\alpha t^{2}}{2}=\frac{\gamma \tau^{2}}{2}, \text { for } \Phi_{0}=0 \\
& \xi=\frac{g \bar{\xi}}{\omega_{n}^{2}}, \quad \eta=\frac{g \bar{\eta}}{\omega_{n}^{2}}, \quad \dot{\xi}=\frac{g \bar{\xi}^{\prime}}{\omega_{n}}, \quad \dot{\eta}=\frac{g \bar{\eta}^{\prime}}{\omega_{n}} \\
& \ddot{\xi}=g \bar{\xi}^{\prime \prime}, \quad \ddot{\eta}=g \bar{\eta}^{n}
\end{aligned}
$$

where

$$
\begin{aligned}
& (\cdot)^{\prime}=\frac{d(\cdot)}{d \tau}=\frac{1}{\omega_{n}} \frac{d(\cdot)}{d t}, \quad(\cdot)^{\prime \prime}=\frac{d^{2}(\cdot)}{d \tau^{2}}=\frac{1}{\omega_{n}^{2}} \frac{d^{2}(\cdot)}{d t^{2}} \\
& \theta=\Phi+\beta ; \quad \psi=\Phi-\arctan \left(\frac{y}{z}\right) \text { (see Fig. 1(b)). }
\end{aligned}
$$

\section{RESULTS AND DISCUSSION}

The presence of a crack on the shaft results in the stiffness variation as a function of time. In most rotor dynamic analysis, the system response is obtained in the form of steady state amplitude data at each operational speed. In the case of transient analysis, the dynamic response can be obtained using time marching techniques. In this study Runge-Kutta method was employed to numerically solve equations of motion (Eq. (7a) and (7b)). The nonlinear responses of rotor passing through the critical speed with several values of constant angular acceleration ratios $\left(\gamma=\frac{\alpha}{\omega_{n}^{2}}\right)$ and different 
crack depths $(\Delta K)$ were evaluated. For all cases it was assumed that damping ratio $\zeta=0.05$ and normalized unbalance eccentricity is $\frac{\varepsilon}{\eta_{s t}}=0.05$.

The change in the rotor phase response due to the acceleration and crack is presented in Fig. 4. In general, due to the crack the amplitude of the phase angle decreases for the given speed ratio and acceleration rate. In addition, it can be seen that the crack induces significant oscillations in the rotor phase response (see Fig. (4b)), especially for higher acceleration rates. Such a characteristic "saw-cut" pattern of the phase waveform can find potential application in the crack on-line detection monitoring systems.

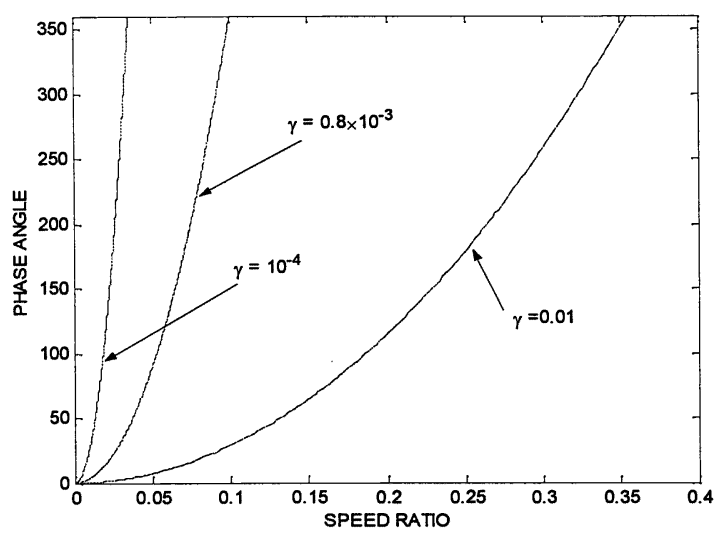

(a) no crack

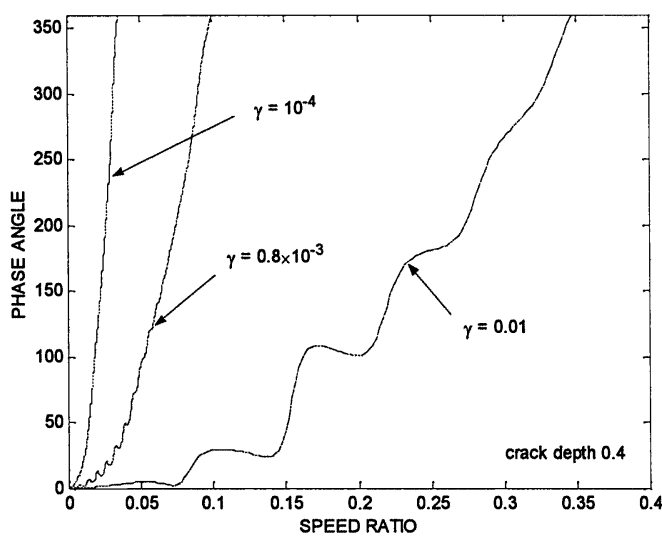

(b) $\Delta K=0.4$

Figure 4: Phase angle of the accelerated rotor passing through the critical speed for $\gamma=0.01,0.8 \times 10^{-3}$, and $10^{-4}$, and $\beta=0$.

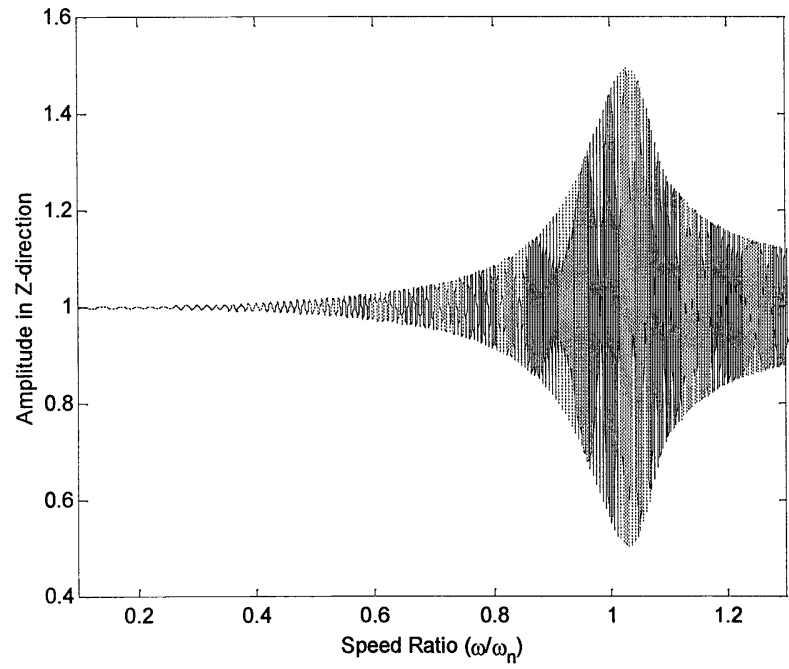

(a) no crack

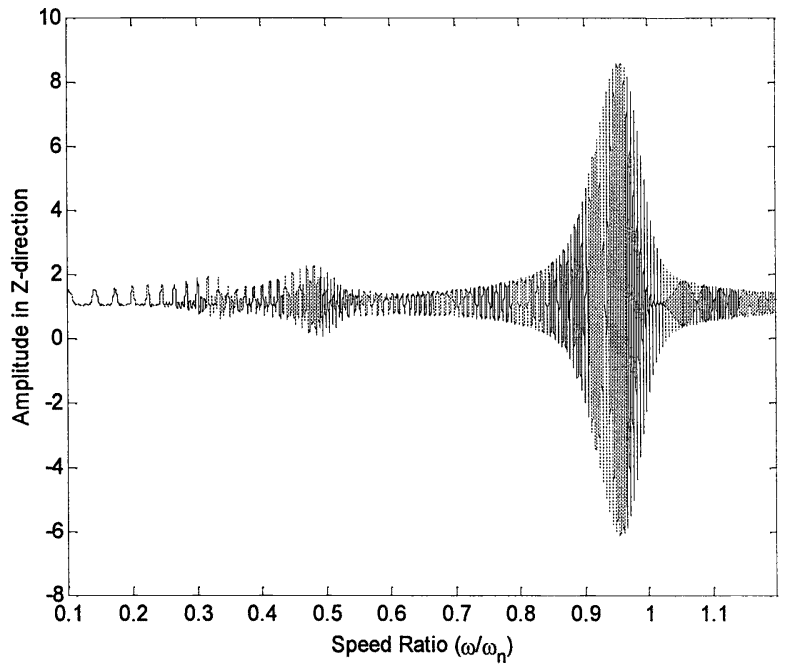

(b) $\Delta K=0.32$

Figure 5: Vibration amplitude in Z-direction of the accelerated rotor passing through the critical speed for $\gamma=0.8 \times 10^{-3}$ and $\beta=0$. 


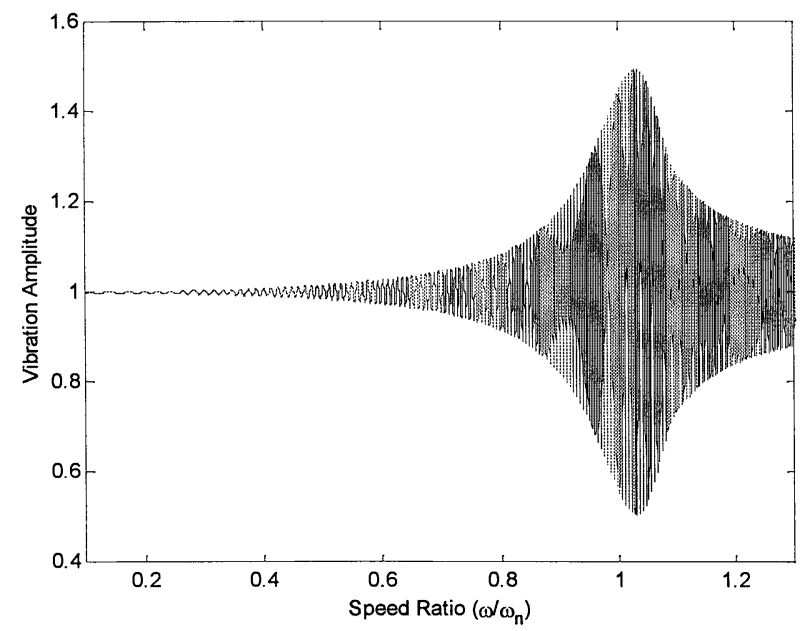

(a) no crack

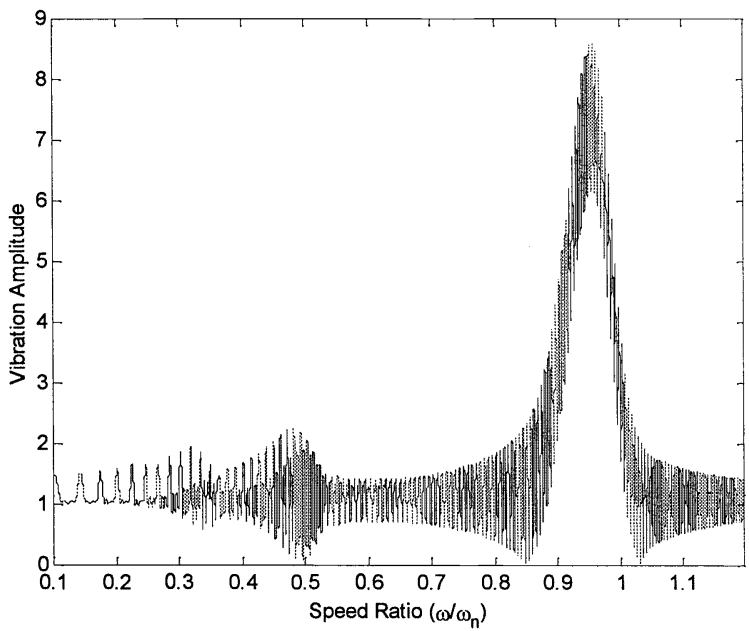

(b) $\Delta K=0.32$

Figure 6: Vibration amplitude of the accelerated rotor passing through the critical speed for $\gamma=0.8 \times 10^{-3}, \beta=0$ and $\Delta K=0.32$.

Figures 5 and 6 show nondimensional vibration amplitude in Z-direction and the overall amplitude, respectively, for the crack depth of $\Delta K=0.32$. It is apparent that the shaft crack reduces the stiffness of the system, shifting the resonance to a lower speed. It can be noticed the presence of sub-critical $(1 / 3,1 / 2)$ response peaks and large increase of vibration response at the fundamental resonance peak when the crack exists.

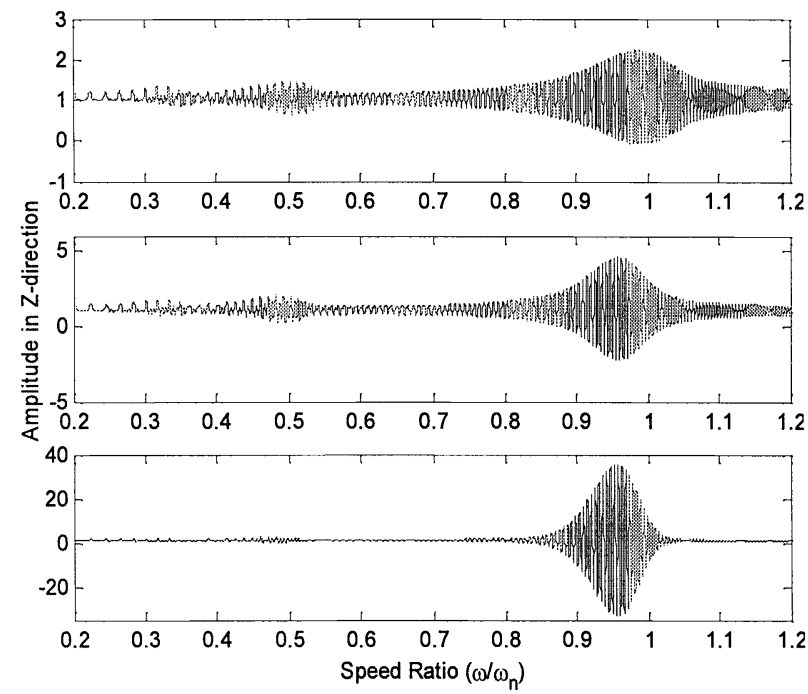

(a)
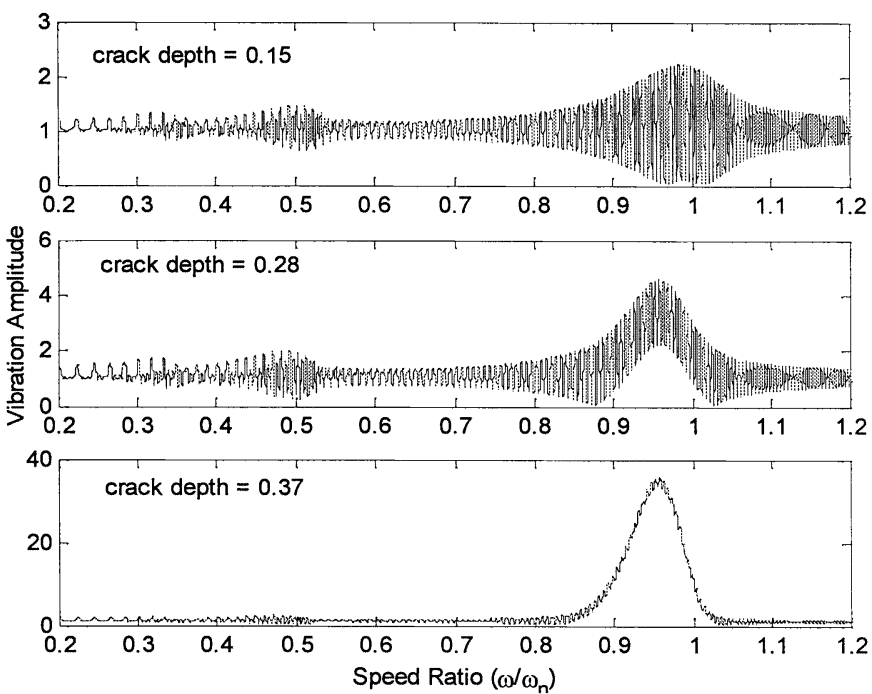

(b)

Figure 7: Response of accelerated rotor passing through the critical speed with crack and gravity for $\gamma=0.8 \times 10^{-3}, \beta=0$ and $\Delta K=$ $0.15,0.28$, and 0.37 ; (a) vibration amplitude in $Z$-direction, (b) vibration amplitude.

The vibration amplitude in Z-direction and the overall vibration amplitude transient responses for different crack depths and constant acceleration rate are illustrated in Fig. 7. It can be noticed (see Fig. 7(b)) that the whirl vector develops significant oscillations near the fundamental resonance speed. The zone of critical speed becomes wider and the vibrations level increases with the depth of the crack. As a result, for the deeply cracked rotors there is no exact critical speed. 
Presentation of the rotor transient response in rotating coordinates also provides useful information. Thus, Fig. 9 shows vibration amplitude in $\xi$-direction of the accelerated rotor passing through the critical speed for crack depths $\Delta K=0.32$ and 0.36 . In the absence of the crack the resonant vibration amplitude almost does not show-up. However, if there is a crack then the fundamental resonance is apparent and additionally the second and third harmonics are present, which is a crack-characteristic phenomenon. It can be seen that even small change in the crack depth causes that the rise of amplitude.

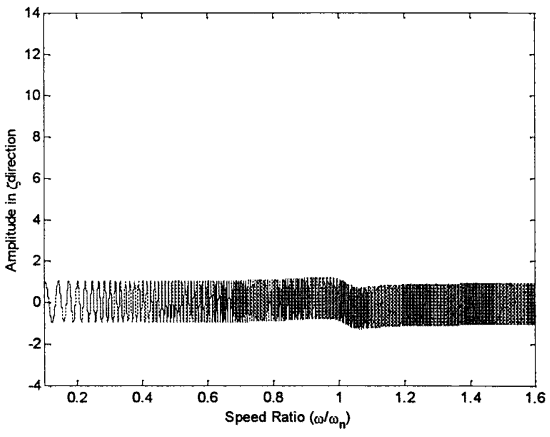

(a)

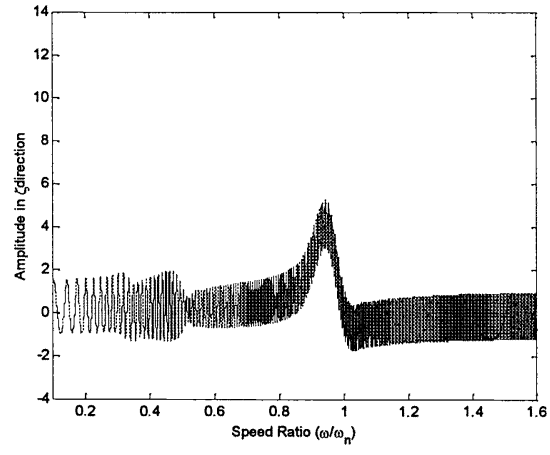

(b)

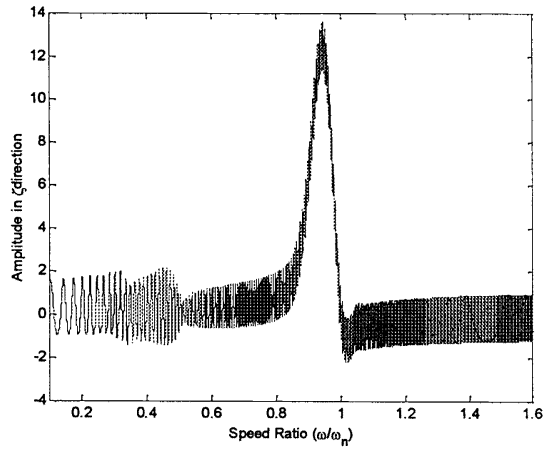

(c)

Figure 9: Vibration amplitude in $\xi$-direction of the accelerated rotor passing through the critical speed for $\gamma=0.8 \times 10^{-3}$ and $\beta=0$; (a) no crack, (b) $\Delta K=0.32$, (c) $\Delta K=0.36$.

The effect of unbalance eccentricity orientation on vibration amplitude of a cracked rotor is shown in Fig. 10. Orientation of unbalance affects the amplitude of the fundamental resonance, but does have a little influence on the subharmonics. The maximum resonant vibration amplitude exists when the eccentricity vector is along the crack centerline $(\beta=0)$. When the unbalance is on the opposite side of the crack $\left(\beta=180^{\circ}\right)$, then the effect of the crack is significantly diminished. In this situation, the resonance amplitude is only about $1 / 6$ of the corresponding amplitude for the case when $\beta=0$.
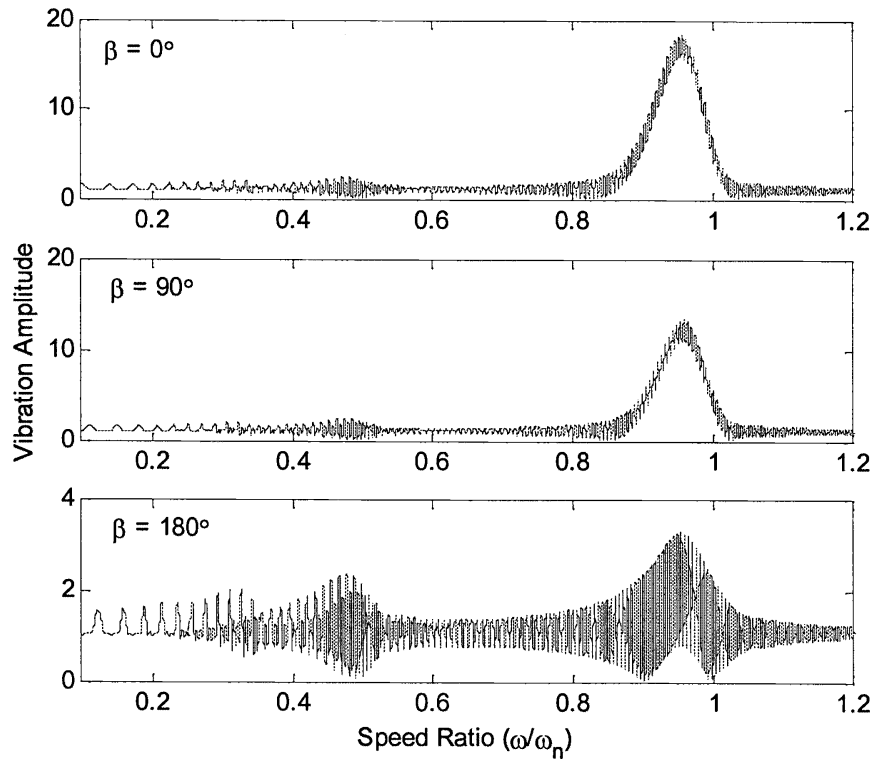

Figure 10: The effect of eccentricity orientation on vibration amplitude of a cracked rotor for $\beta=0^{\circ}, 90^{\circ}$ and $180^{\circ}$, $\Delta K=0.35$, and $\gamma=0.8 \times 10^{-3}$. 


\section{CONCLUSIONS}

The presence of a crack in the shaft of the rotor system affects the dynamic response significantly. The changes of vibrational behavior can be used to predict and/or detect the crack in the shaft. The following conclusions can be drawn based on the results presented in this paper:

1. The developed model enables to study the cracked rotor dynamic response with and without weight dominance. The model includes small and deep cracks, as well as cross stiffness effect (for deep cracks).

2. Characteristics "saw-cut" pattern in the phase response waveform can find potential application in the on-line crack detection monitoring systems.

3. The crack reduces stiffness of the system, shifting the resonance to lower speeds. This effect is magnified by the depth of the crack. It can be noticed the presence of subcritical $(1 / 3,1 / 2)$ response peaks and large increase of fundamental vibration response when the crack exists.

4. For the accelerating rotor the zone of critical speed becomes wider and the vibration level increases with the depth of the crack. The whirl vector develops oscillation near the critical speed.

5. The orientation of unbalance eccentricity with respect to the crack centerline affects fundamental resonance and does have much less effect on subharmonics.

\section{NOMENCLATURE}

$\begin{array}{ll}C & \text { external damping coefficient } \\ g & \text { gravitational acceleration } \\ K & \text { uncracked shaft stiffness } \\ K_{\xi} & \text { cracked shaft stiffness in } \xi \text {-direction } \\ K_{\eta} & \text { cracked shaft stiffness in } \eta \text {-direction } \\ M & \text { mass of the disk } \\ O_{b} & \text { position of bearing centers line } \\ O_{s} & \text { shaft center } \\ z, y, Z, Y & \text { inertial coordinate system; } Z=z / \eta_{s t}, Y=y / \eta_{s t} \\ \alpha & \text { constant angular acceleration } \\ \beta & \text { angle between } \xi \text { and unbalance } \\ \gamma & \text { acceleration ratio; } \gamma=\alpha / \omega_{n}^{2} \\ \Delta K & \text { stiffness change ratio }\left(=\Delta K_{\xi} / K \text { ) }\right. \\ \Delta K_{\xi} & \text { variation of stiffness in } \xi \text {-direction caused by crack } \\ \Delta K_{\eta} & \text { variation of stiffness in } \eta \text {-direction caused by crack } \\ \varepsilon & \text { unbalance eccentricity of the disk } \\ \zeta & \text { external damping ratio } \\ \eta_{s t} & \text { static deflection of uncracked rotor; } \eta_{s t}=g / \omega_{n}^{2} \\ \theta & \text { orientation of unbalance eccentricity with respect to and } z \text {-axis } \\ \theta_{r} & \text { whirling angle corresponding to whirling speed } \\ \xi, \eta & \text { rotating coordinate system; } \xi \text { is in the crack direction } \\ \psi & \text { angle between } \xi \text { and the line connecting bearing and shaft center } \\ \Phi & \text { angle between } \xi \text { and } z \text { axes } \\ \omega & \text { angular velocity of rotation } \\ \omega_{n} & \text { critical speed of uncracked rotor }\end{array}$




\section{REFERENCES}

1. Bently Nevada, "Early Shaft Crack Detection on Rotating Machinery Using Vibration Monitoring and Diagnostics," Technical Bulletin No. L1036-00, No. 1, 1986.

2. I. Imam, et al., "Development of an On-Line Crack Detection and Monitoring System," ASME Journal of Vibration, Acoustics, Stress, and Reliability in Design, 111, pp. 241-250, 1989.

3. F.M. Dimentberg, "Flexural Vibration of Rotating Shafts," Butterworth, London, 1961.

4. T.A. Henry and B.E. Okah-Avae, "Vibrations in Cracked Shafts," Paper C162/76, Proc. of I.Mech.E. Conference on Vibrations in Rotating Machinery, pp. 15-19, 1976.

5. I.W. Mayes and W.G.R. Davies, "The Vibrational Behaviour of a Rotating Shaft System Containing a Transverse Crack," Paper C168/76, Proc. of I.Mech.E. Conference on Vibrations in Rotating Machinery, pp. 53-64, 1976.

6. I.W. Mayes and W.G.R. Davies, "Analysis of the Response of a Multi-Rotor-bearing System Containing a Transverse Crack in a Rotor," ASME Journal of Vibration, Acoustics, Stress, and Reliability in Design, 106, pp 139-145, 1984.

7. R. Gasch, "Dynamic Behavior of a Simple Rotor with a Cross-sectional Crack", Paper C178/76, Proc of I.Mech.E. Conference on Vibrations in Rotating Machinery, pp. 123-128, 1976.

8. R. Gasch, "A Survey of the Dynamic Behavior of a Simple Rotating Shaft with a Transverse Crack," Journal of Sound and Vibration, 160(2), pp. 313-332, 1993.

9. B. Grabowski, "The Vibrational Behavior of a Turbine Rotor Containing a Transverse Crack," ASME Journal of Mechanical Design, Vol. 102, pp. 140-146, 1980.

10. Sawicki, J.T., "Some Advances in Diagnostics of Rotating Machinery Malfunctions," Invited Paper, Proc. of International Symposium on Machine Condition Monitoring and Diagnosis, The Japan Society of Mechanical Engineers, pp. 138-144, 2002.

11. Federal Aviation Administration, "Rules Docket No. 2001-SW-01-AD," Federal Register, March 2, 2001.

12. J. Wauer, "On the Dynamics of Cracked Rotors: A Literature Survey," Applied Mechanics Review, 43(1), pp. 13-17, 1990.

13. R.H. Plaut, R.H. Andruet, and S. Suherman, "Behavior of a Cracked Rotating Shaft During Passage Through a Critical Speed," Journal of Sound and Vibration, 173(5), pp. 577-589, 1994.

14. A.S. Sekhar and B.S. Prabhu, "Transient Analysis of Cracked Rotor Passing Through Critical Speed," Journal of Sound and Vibration, 173(3), pp. 415-421, 1994.

15. A.S. Sekhar and B.S. Prabhu, "Condition Monitoring of Cracked Rotors Through Transient Response," Mechanism and Machine Theory, 33(8), pp. 1167-1175, 1998. 